\title{
Interactive comment on "Fennec dust forecast intercomparison over the Sahara in June 2011" by J.-P. Chaboureau et al.
}

\section{Anonymous Referee \#2}

Received and published: 14 April 2016

\section{General Comments:}

The paper presents a dust modeling intercomparison against measurements performed during Fennec campaign in June 2011. The authors focus on the ability of four limited area models (ALADIN, AROME, Meso-NH20km, Meso-NH5km) to forecast atmospheric and dust properties during the campaign, performed over the western Sahara. They claim that this is to be "the first ever intercomparison of dust forecasts over the western Sahara". Obviously this is not true since a big number of dust model evaluation studies as well as operational products for this area are already available based on comparisons with satellite and AERONET data (e.g. SDS-WAS). The possible added value in this work would come from a potential use of the field aircraft measurements for model evaluation. This is partially done in this paper but a larger part of the work is devoted on comparisons with satellites and AERONET.

Printer-friendly version

Discussion paper 
Furthermore, I am concerned about the low modeling skill that is found in this study which may be due to the inability of the specific models to reproduce the atmospheric ACPD dust processes or due to improper configuration of the simulations.

Therefore I would recommend the publication of this paper, after the authors proceed with some major revisions that include:

1. Rerunning the models to homogenize initial conditions, domain setups and increase the forecasting horizon for the high resolution runs.

2. Updating satellite products used for the evaluation (e.g. MODIS collection 6, including DB for Terra)

3. Moreover I would suggest that the authors will focus also on the in-situ data provided for specific case studies of particular interest by Ryder et al. (2015). Such analysis could provide more insight on the capabilities and restrictions of each model in reproducing the dust related processes.

Details on the aforementioned suggestions are given below along with other minor comments.

Specific Comments:

Line 16: Please replace extinction with concentrations

Line 53: Two aircrafts

Line 91: centred - centered

Line 92: What is a "piecewise parabolic method advection scheme" ?

Line 103: Please give more information on ARPEGE model

Printer-friendly version

Line 103: There is no use in comparing models with different initial conditions. Even the same model will produce different results when initialized at different times.

Discussion paper

Line 106: Don't you assume any model spin up time for Meso-NH? By initializing the

C2 
model every 24 hours you are most probably just interpolating ECMWF data on your domain rather than allowing the higher resolution model to develop its own atmospheric fields.

Lines 100-115: Please give also information for the vertical model grid structure.

Lines 137-140: What is the physical meaning of this tuning factor?

Interactive

comment

Line 162: "In general, dust is characterized by low values of Ångstrom exponent, less than 0.4". Did you filter out AOD measurements with Angstrom > 0.4?

Lines 169-172 : Do you consider using updated MODIS DB product from Collection 6 for your comparisons throughout the paper? And please check if Terra DB product is available already.

Line 175: "63\% of the MISR AODs fell within 0.05 or $20 \%$ of AERONET AODs". This phrase is not clear - please explain further.

Lines 233 - 307: Comparison between models and AERONET in section 3.2 indicate a very low modeling skill which makes the analysis presented in the following sections questionable.

Line 297: "There was no strong contrast in scores between forecasts initialized with ARPEGE and ECMWF nor in forecasts at low and high resolutions". How do you explain this taking also into consideration the model differences mentioned in lines 309-315?

Lines 314-315. I would strongly recommend to run again AROME for Fennec period extending also the modeling domain so that it is comparable to ALADIN.

Lines 334-335: Why not attributed to LLJ?

Printer-friendly version

Line 340: "Here, the ITD was defined as the southern limit where the mixing ratio of water vapour at $2 \mathrm{~m}$ equals $10 \mathrm{~g} \mathrm{~kg}-1$ ". At which model? 
Line 355: Please examine if these are indeed cold pools and explain how they are defined in the models.

Line 372-373 : Not clear - Please rephrase.

Lines 410-420: What is the correlation between the wind regimes and dust episode severity? For example if the $6 \%$ cold pool corresponds to one or two episodes during the 1 month period this could be a significant contribution.

Interactive

comment

Lines 457-458: How do you define "rather well" and "remarkably well"? Please use statistical metrics.

Lines 545-546: "AROME was initialized at 18:00UTC the previous day and was able to forecast the development of the thunderstorms over the Atlas Mountains and the associated cold pools (not shown)". This is an important finding and it is definitely worth showing.

Lines 549-550: "However, the meteorological imprint of the associated density currents was removed when initializing the Meso-NH forecasts with ECMWF 00:00UTC analysis on 21 June." Following my previous comment in Line 106 I would strongly recommend that you run again at least Meso-NH $5 \mathrm{~km}$ for the Fennec period but this time including a sufficient spin up time.

Line 593: "the high-resolution models performed better than the low-resolution ones". I guess that we know that already.

Line 603: "At daily time scales, the vertical structure of temperature and humidity was forecast well“. How do you define well? Please use statistical metrics.

Line 605: "All the models forecast dust extinction within the SABL correctly". So why do you multiply ALADIN emission by a factor of 3 ?

Interactive comment on Atmos. Chem. Phys. Discuss., doi:10.5194/acp-2016-12, 2016. 